\section{Basal cell carcinoma developed from an epidermal cyst: A case report and review of the litera- ture}

\author{
Tomoka Harada, ${ }^{1,2}$ Takeshi Fukumoto, ${ }^{1}$ \\ Hideki Shimizu, ${ }^{2}$ Chikako Nishigori ${ }^{1}$ \\ ${ }^{1}$ Division of Dermatology, Department of \\ Internal Related, Kobe University \\ Graduate School of Medicine, Kobe; \\ ${ }^{2}$ Division of Dermatology, Sanda City \\ Hospital, Sanda, Japan
}

\begin{abstract}
Cutaneous epidermal cysts are common benign cysts derived from the epidermis or epithelium of hair follicles, and malignancy originating from epidermal cysts is uncommon. When a cutaneous epidermal cyst turns malignant, it is mostly squamous cell carcinoma, and basal cell carcinoma is rare.

We present the case of a 58-year-old man with basal cell carcinoma originating from an epidermal cyst on the buttocks. Histopathological analysis with hematoxylin-eosin (H\&E) staining showed the presence of the cyst, the wall of which was partially replaced by a malignant tumor. Moreover, the cyst was filled with malignant tumor cells in some areas. The tumor cells were basaloid, and extended through the dermis down to the subcutaneous tissue in a solid pattern. Immunoperoxidase staining for Ber-EP4 was positive.

To our knowledge, there are 11 reported cases of BCC originating from epidermal cysts in English, and we reviewed these reports and examined potential trends. We estimate that some longstanding epidermal cysts may have the potential of malignant transformation, and chronic and repeated irritation might trigger malignancy from epidermal cysts. We emphasize that benignlooking cystic lesions showing progressive growth should be examined histopathologically.
\end{abstract}

\section{Introduction}

Cutaneous epidermal cysts are common benign disorders derived from the epidermis or epithelium of hair follicles, and their malignant transformation is uncommon. ${ }^{1-9}$ Bauers et al. reported that $2.2 \%$ of cases degenerate into malignant tumors; namely, 72 carcinomas were found in 3,300 cysts. ${ }^{1}$ Squamous cell carcinoma (SCC) occurred in more than $90 \%$ of these malignant cases and basal cell carcinoma (BCC) was identified in the remaining cases; this shows the rare incidence of $\mathrm{BCC}$ originating from cysts. ${ }^{1}$ In addition, Tanaka et al. described $\mathrm{BCC}$ as the second most common type of cancer originating from epidermal cysts, with an overall rate of approximately $0.1 \% .^{5}$ Herein, we present a rare case of $\mathrm{BCC}$ arising from an epidermal cyst on the buttocks. To our knowledge, only 11 such cases have been reported in English, and we reviewed them (Table 1). ${ }^{2-10}$

\section{Case Report}

A 58-year-old man noticed a subcutaneous mass on his right buttocks that appeared 2 years before, and recently the lesion rapidly grew to a diameter of $3 \mathrm{~cm}$ within 2 months. As the mass ruptured, the patient visited the emergency department, and an open drainage was performed. After 1 week, he visited our hospital, and we noticed a subcutaneous induration on the right buttocks, with slight pigmentation (Figure 1a). Computed tomography imaging showed a subcutaneous well-demarcated oval-shaped mass located on the right buttocks, measuring $4 \times 3 \mathrm{~cm}$ (Figure $1 \mathrm{~b}$ ) in size. Ultrasound examination showed a subcutaneous hypo-echoic mass (Figure 1c). The lesion presented non-homogeneous internal echo-brightness with enhanced posterior echo and slight blood flow signals (Figure 1c). An incisional biopsy was performed, and the histopathologic analysis with hematoxylin-eosin staining showed the presence of the cyst, the wall of which was partially replaced by malignant cells (Figure 1d). The overlying skin showed no remarkable changes. Moreover, the cyst was filled with malignant cells in some areas (Figure 1d, e, and g). The tumor cells were basaloid and extended through the dermis down to the subcutaneous tissue in a solid pattern (Figure 1d).

The cells in the rim of the tumor nests showed a palisading arrangement of the nuclei (Figure 1g) and the tumor cells were positive for Ber-EP4 staining (Figure $1 \mathrm{f}$ and h). Based on the histological findings, we diagnosed this tumor as a $\mathrm{BCC}$ originating from an epidermal cyst. A wide excision was performed on the fascia with $1 \mathrm{~cm}$ margins from the tumor, and there was no evidence of recurrence on examination after 2 years and 3 months.

\section{Discussion and Conclusions}

Although BCC arising from epidermal
Correspondence: Takeshi Fukumoto, Division of Dermatology, Department of Internal Related, Kobe University Graduate School of Medicine, 7-5-1 Kusunoki-cho, Chuo-ku, Kobe 650-0017, Japan.

Tel.: +81783826134 - Fax: +81783826149 .

E-mail: fuku@med.kobe-u.ac.jp

Key words: Basal cell carcinoma, buttocks, cutaneous cyst, epidermal cyst, malignant transformation.

Contributions: The authors contributed equally.

Conflict of interest: The authors declare no potential conflict of interest.

Funding: None.

Consent to publication: The patient whose case is presented in this manuscript has given written informed consent to publish his case details.

Received for publication: 22 May 2021

Accepted for publication: 25 May 2021

This work is licensed under a Creative Commons Attribution-NonCommercial 4.0 International License (CC BY-NC 4.0).

${ }^{\circ}$ Copyright: the Author(s), 2021

Licensee PAGEPress, Italy

Dermatology Reports 2021; 13:9273

doi:10.4081/dr.2021.9273

cyst is very rare and it may be difficult to suspect BCC from subcutaneous induration, ${ }^{1-9}$ benign-looking cystic lesions showing progressive growth should be examined histopathologically. A review of 12 similar cases revealed that eight cases $(67 \%)$ were identified in locations other than the face, despite the fact that most BCCs are on the face. They were located in the shoulder, knee, neck, chest, back, and buttocks, which are susceptible to mechanical irritation and load. Thus, it is reasonable to speculate that these chronic and repeated irritations might trigger malignant transformation of cells in the wall of epidermal cysts.

Moreover, as suggested previously, ${ }^{3,5}$ our review revealed an interesting trend, that is, long-term presence of an epidermal cyst before the development of BCC; this suggests that some longstanding epidermal cysts may be a risk of the development of malignant tumors. Further studies are needed to clarify the underlying pathomechanism; however, our report adds new information regarding this coexistence. 
Table 1. Clinical findings of published cases and the present case of basal cell carcinoma occurring in an epidermal cyst.

\begin{tabular}{|c|c|c|c|c|c|c|c|c|}
\hline Case & Ref. & Age & Gender & Location & ize of tumor ( $\mathrm{mm})$ & Clinical description & Period & Tissue type \\
\hline 1 & {$[2]$} & 65 & $\mathrm{~F}$ & Shoulder & Unknown & Subcutaneous mass & Several years & Unknown \\
\hline 2 & {$[3]$} & 78 & M & Upper eyelid & $15 \times 12-22 \times 8$ & Nodular lesion & 50 years & Solid \\
\hline 3 & {$[7]$} & 40 & $\mathrm{~F}$ & Chest & $20-30$ & Subcutaneous nodule & years & Solid \\
\hline 4 & [7] & 31 & M & Neck & Unknown & Nodular lesion & Unknown & Solid and adenoid \\
\hline 5 & {$[4]$} & 55 & M & Back & 20 & Subcutaneous mass & 20 years & Unknown \\
\hline 6 & {$[5]$} & 76 & M & Knee & $45 \times 25$ & Subcutaneous mass & 15 years & Solid and adenoid \\
\hline 7 & {$[10]$} & 72 & $\mathrm{~F}$ & Lower eyelid & $3 \times 2 \times 1$ & Whitish nodule & 2 months & Unknown \\
\hline 8 & {$[8]$} & 50 & M & Shoulder & 35 & Subcutaneous mass & Long years & Nodulocystic \\
\hline 9 & {$[6]$} & 59 & M & Back of neck & Unknown & Cystic lesion & Unknown & Superficial, nodular \\
\hline 10 & {$[6]$} & 55 & $\mathrm{M} \mathrm{F}$ & Frontal region of head & Unknown & Cystic lesion & Unknown & Unknown \\
\hline 11 & {$[9]$} & 46 & $\mathrm{~F}$ & Nose & $10 x 10 \times 8$ & Blackish mass & Unknown & Unknown \\
\hline $12 \operatorname{Pr}$ & sent case & 58 & M & Buttocks & $40 x 30$ & Subcutaneous mass & 2 years & Solid \\
\hline
\end{tabular}

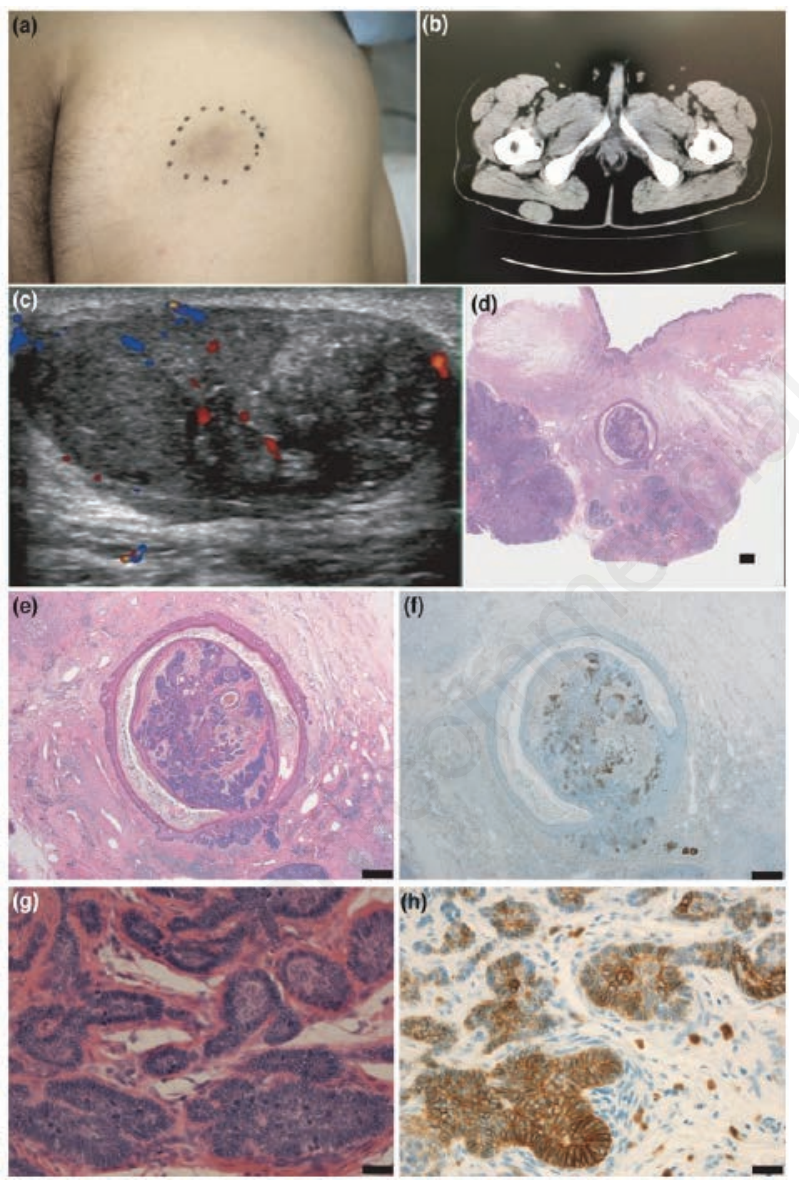

Figure 1. Clinical and histopathological features of the patient. (a) Pigmented lesion and subcutaneous induration on the right buttocks. (b) Computed tomography image of the axial section showing a subcutaneous mass on the right buttocks. (c) Ultrasound image showing a subcutaneous, non-homogeneous hypo-echoic mass with enhanced posterior echo and blood flow signals. (d, e) Histopathological analysis showing cysts in the dermis. The wall of the cyst was lined with stratified squamous epithelium exhibiting keratinization similar to the epidermis. The cyst was filled with horny material and malignant tumor, and the tumor extended through the dermis down to the subcutaneous tissue in a solid pattern. Hematoxylin and eosin $(H \& E)$ staining, scale bar $=600 \mu \mathrm{m}[\mathrm{d}]$, original magnification: $\times 20$; scale bar $=500 \mu \mathrm{m}[\mathrm{e}]$. (f) Positive reaction for Ber-EP4 staining in tumor cells. Original magnification: $\times 20$; scale bar $=500 \mu \mathrm{m}$. $(\mathrm{g})$ The peripheral cell layer of the tumor nests showed a palisading arrangement of the nuclei and clefting artifact between the epithelium and the stroma. H\&E staining, original magnification: $\times 400$; scale bar $=20 \mu \mathrm{m}$. (h) Positive reaction for Ber-EP4 staining in tumor cells. Original magnification: $\times 400$; scale bar $=20 \mu \mathrm{m}$.

\section{References}

1. Bauer BS, Lewis VL, Jr. Carcinoma arising in sebaceous and epidermoid cysts. Ann Plast Surg 1980;5:222-6.

2. Delacretaz J. Keratotic basal-cell carcinoma arising from an epidermoid cyst. J Dermatol Surg Oncol 1977;3:310-1.

3. Ikeda I, Ono T. Basal cell carcinoma originating from an epidermoid cyst. J Dermatol 1990;17:643-6.

4. Dini M, Innocenti A, Romano GF. Basal cell carcinoma arising from epidermoid cyst: a case report. Dermatol Surg 2001;27:585-6.

5. Tanaka M, Terui T, Sasai S et al. Basal cell carcinoma showing connections with epidermal cysts. J Eur Acad Dermatol Venereol 2003;17:581-2.

6. Udovenko O, Guo Y, Connelly T et al. Basal-Cell Carcinoma Occurring in Cutaneous Infundibular Cysts: Report of 2 Cases and Review of the Literature. Am J Dermatopathol 2015;37:635-8.

7. Mehregan DA, al-Sabah HY, Mehregan AH. Basal cell epithelioma arising from epidermoid cyst. J Dermatol Surg Oncol 1994;20:405-6.

8. Liau JL, Altamura D, Ratynska M et al. Basal cell carcinoma arising from an epidermal cyst: when a cyst is not a cyst. Case Rep Dermatol 2015;7:75-8.

9. Terada T. Basal cell carcinoma arising from epidermal cyst. J Cutan Med Surg 2015;19:105-6.

10. Jakobiec FA, Zakka FR, Hatton MP. Eyelid basal cell carcinoma developing in an epidermoid cyst: a previously unreported event. Ophthalmic Plast Reconstr Surg 2010;26:491-4. 